\title{
Fundamental Transverse Mode Selection (TMS\#0) of Broad Area Semiconductor Lasers with Integrated Twice-Retracted $4 f$ Set-Up and Film-Waveguide Lens
}

\author{
Henning Fouckhardt, Ann-Kathrin Kleinschmidt, \\ Johannes Strassner, and Christoph Doering
}

Integrated Optoelectronics and Microoptics Research Group, Physics Department, University of Kaiserslautern, P.O. Box 3049, 67653 Kaiserslautern, Germany

Correspondence should be addressed to Henning Fouckhardt; fouckhar@physik.uni-kl.de

Received 4 September 2017; Accepted 22 November 2017; Published 14 December 2017

Academic Editor: Vasily Spirin

Copyright (C) 2017 Henning Fouckhardt et al. This is an open access article distributed under the Creative Commons Attribution License, which permits unrestricted use, distribution, and reproduction in any medium, provided the original work is properly cited.

Previously we focused on fundamental transverse mode selection (TMS\#0) of broad area semiconductor lasers (BALs) with two-arm folded integrated resonators for Fourier-optical spatial frequency filtering. The resonator had a round-trip length of $4 f$, where $f$ is the focal length of the Fourier-transform element (FTE), that is, a cylindrical mirror in-between the orthogonal resonator branches. This $4 f$ set-up can be called "retracted once" due to the reflective filter after $2 f$; that is, the $2 f$ path was used forwards and backwards. Now the branches are retracted once more resulting in a compact $1 f$ long linear resonator (called "retracted twice") with a roundtrip length of $2 f$. One facet accommodates the filter, while the other houses the FTE, now incorporating a film-waveguide lens. The BAL facet with the filter represents both the Fourier-transform plane (after $2 f$, i.e., one round-trip) as well as the image plane (after $4 f$, two round-trips). Thus filtering is performed even after $4 f$, not just after $2 f$. Experimental results reveal good fundamental TMS for pump currents up to $20 \%$ above threshold and a one-dimensional beam quality parameter $M_{1 \mathrm{D}}^{2}=1.47$. The BALs are made from AlGaInAsSb, but the concept can equally well be employed for BALs of any material system.

\section{Introduction}

Broad area (semiconductor diode) lasers (BALs) allow for the generation and extraction of higher light output powers as compared to conventional narrow stripe lasers. The higher power for larger pump currents is distributed over a larger cross-section thus avoiding catastrophic optical mirror damage (COMD). Typical BALs exhibit emitter widths of $50-200 \mu \mathrm{m}$. The drawback is represented by the build-up and oscillation of larger transverse modes (in cases without further provisions) limiting the portion of the higher light power easily focused into a small volume or onto a small area.

Therefore, techniques have to be employed, which are suitable for support of the fundamental transverse mode (mode \#0) by suppression of higher order modes upon laser oscillation build-up. In most cases either the BAL facets or the lateral (effective) refractive index or pump current distributions are modified [1-8] (if so including transverse Bragg gratings $[7,8])$ or an external cavity is used [7-14] to achieve the desired transverse mode selection (TMS) (and if applicable also to stabilize a longitudinal mode [7, 8]). In all these cases eventually spatial frequency filtering is performed. (Feedback from external cavities might also destabilize the BAL emission [15-19], e.g., leading to self-pulsation.) Also approaches with tapered lasers or laser-tapered amplifier arrangements or a current injection region narrower than the active region are known [20-23].

Low spatial frequencies stand for low transverse mode numbers and vice versa. Thus, in order to achieve fundamental mode TMS a low-pass filter has to be applied.

\section{Concepts}

Formerly we have integrated a Fourier-optical $4 f$ set-up into the semiconductor resonator with two orthogonal branches 


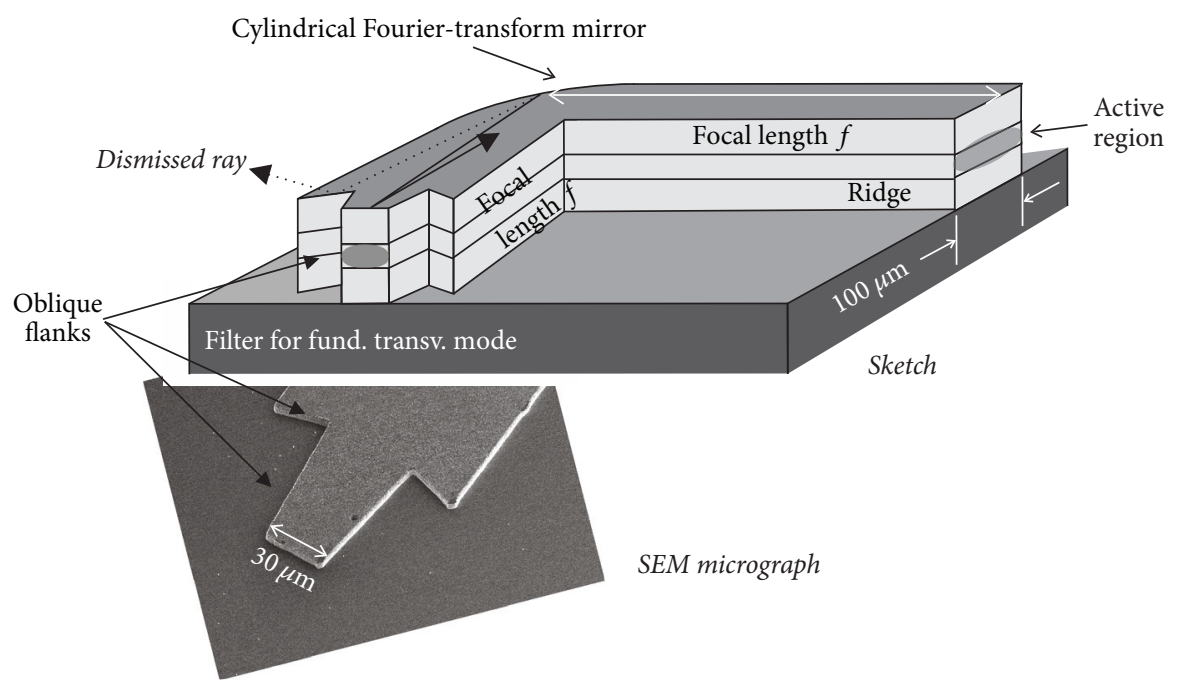

FIGURE 1: Former concept ( $4 f$ set-up "retracted once"): sketch of a BAL laser with integrated $4 f$ set-up in the configuration with two orthogonal branches. The spatial filter to support the fundamental mode is made up of a nontilted and several tilted/oblique flanks/segments, the latter intended to refract and reflect unwanted rays out of the resonator and to reflect the supported rays back into the resonator. Fresnel reflectivity of $\approx 31 \%$ at the semiconductor-air interface is used. In the bottom part of the figure there is an SEM (scanning electron microscope) image of the facet with filter of an actual TMS\#0-BAL sample.

$[24,25]$, as sketched in Figure 1. The resonator length has been $2 f$ and the round-trip path length has come up to $2 f$ plus $2 f$ after Fresnel-reflection at the spatial frequency filter. This $4 f$ set-up might be called "retracted once" due to the reflective arrangement. The dry-etched bend in-between branches has been cylindrical and has officiated as reflective Fourier-transform element. One of the resonator facets has been used as the output plane of the laser; the other facet has housed the spatial frequency filter, realized by flanks for redirection of certain groups of light rays.

In detail: rays with low propagation angles with respect to the optical axis account for low spatial frequencies and thus for the fundamental transverse mode $(\# 0)$. They are Fresnelreflected back into the resonator at the semiconductor-air interface of the central (nontilted) filter segment with a reflectivity of about $31 \%$. Rays with larger propagation angles, which correlate with larger spatial frequencies and higher transverse modes, are refracted and reflected out of the resonator by tilted/oblique dry-etched flanks/segments of the spatial filter.

The principle has worked well. But the two-branch resonator has shown some disadvantages like a strong astigmatism due to the $45^{\circ}$ tilt of the Fourier-transform mirror and an overall resonator length of $\approx 5 \mathrm{~mm}$ or more, too long for bestpossible laser efficiency [26]. This statement touches a principle problem of the integration of a $4 f$ set-up: The desired minimum mode width of several $10 \mu \mathrm{m}$, about equal to the central filter segment width of $2 w_{0}$, necessitates a mirror focal length

$$
f=\frac{n_{(\mathrm{eff})} \pi w_{0}^{2}}{\lambda_{0}}
$$

of several millimeters, considering the properties of the 1D spatial Fourier-transform, vacuum wavelength $\lambda_{0}$, and

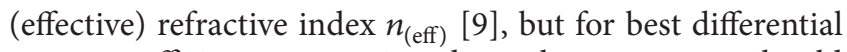
quantum efficiency $\eta$ a semiconductor laser resonator should rather be short [26].

Thus, currently, that is, for this contribution, we have retracted the branches (once more) so that they spatially coincide, giving a compact linear $2 f$ geometry ( $1 f$ plus the path after reflection, the resonator thus called "retracted twice"), as sketched in Figure 2.

One resonator facet contains the low-pass spatial frequency filter to support low spatial frequencies and hence the fundamental transverse mode $(\# 0)$. The other resonator end accommodates the Fourier-transform element.

The BAL facet with the filter represents both the Fouriertransform plane (after $2 f$, i.e., after one round-trip, one Fourier-transformation) as well as the image plane (after $4 f$, two round-trips, both Fourier-transformations).

The laser is an edge-emitting pn diode with intrinsic active region. The $\mathrm{p}$ - and the $\mathrm{n}$-doped regions above and below the active region also serve as the upper and the lower filmwaveguide cladding, respectively. The layer sequence is made from the AlGaInAsSb material system on GaAs substrate and incorporates eight active layers of Stransky-Krastanovgrown GaAsSb quantum dots [27] embedded in GaAs layers. Therefore, the laser emission wavelength is around $933 \mathrm{~nm}$. But the TMS-BAL concept reported here can equally well be utilized for BALs of any material system and emission wavelength.

Ridge lasers are realized, as sketched in more detail in Figure 3 . The laser ridge is $100 \mu \mathrm{m}$ wide, $\approx 2 \mu \mathrm{m}$ high, and $\approx 2.8 \mathrm{~mm}$ long. The central nonoblique segment of the spatial frequency filter is $2 w_{0}=30 \mu \mathrm{m}$ wide. As this segment is considerably narrower than the ridge (some) low-pass spatial filtering is performed even after $4 f$, not just after $2 f$, which is an advantage of this approach for TMS: due to the $30 \mu \mathrm{m}$ 


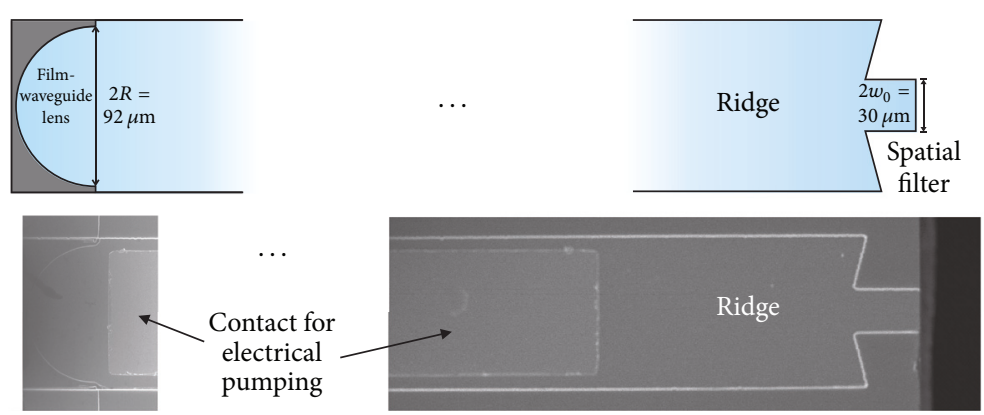

FIGURE 2: Current concept: sketches and SEM micrographs (top-views) of a TMS\#0-BAL with compact linear $f$ long laser resonator ( $4 f$ set-up "retrac ted twice") and spatial filter to support the fundamental transverse mode (\#0). The central filter segment has a width of $2 w_{0}=30 \mu \mathrm{m}$.

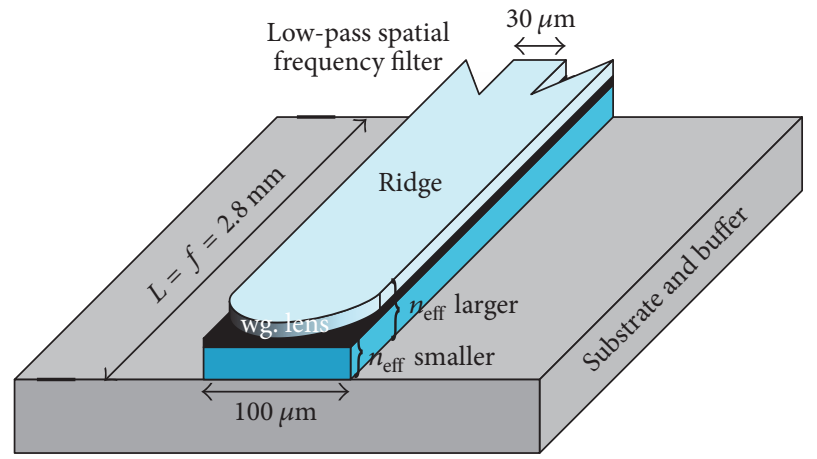

(Doped) claddings

(Intrinsic) active region

FIGURE 3: Current concept again: another sketch (now with oblique view) of a TMS\#0-BAL with compact linear $f$ long laser resonator (4f retracted twice) and spatial filter to support the fundamental transverse mode $(\# 0)$. The dimensions are given and the waveguide lens ("wg. lens") is depicted.

narrow central segment intensity maxima of potentially oscillating higher order transverse modes are stripped, inevitably resulting in suppression of these modes.

The reflective Fourier-transform element is made up of a cleaved plane semiconductor facet in combination with a collecting film-waveguide lens. The latter is realized by dryetching into the upper cladding of the layer sequence stopping $50 \mathrm{~nm}$ below the upper cladding (i.e., within the active region); see also Section 3. This way the effective refractive index $n_{\text {eff }}$ of any laser mode is reduced. (Due to the reduction of the refractive index in the region of the waveguide lens, its top-viewed shape has to resemble that of a diverging lens known from free-space optics in order to achieve a collective function.) Half of the lens function is achieved before reflection of the wave at the plane facet and the other half after this reflection on the wave's way back. The focal length $f$ of the complete (biconcave) film-waveguide lens (with its two plane-concave halves) is supposed to be equal to the resonator length $L(L=f)$.

The radius of curvature $R$ of the waveguide lens is $46 \mu \mathrm{m}$, giving a diameter $2 R$ of $92 \mu \mathrm{m}$, that is, nearly equal to the ridge width (see Figures 3 and 2 again).

\section{Technological Work}

The monocrystalline layer sequences are epitaxially grown with our molecular beam epitaxy (MBE) system R450 by DCA Instruments Oy, Turku, Finland. The central nontilted segment of the spatial frequency filter and the facet on the Fourier element side of the resonator are cleaved. The other (i.e., tilted) flanks are implemented with standard photolithography, using a shared dry-etch step. For the dry-etch processes (reactive ion etching (RIE)) a MicroSys 350 machine by Roth \& Rau, Wuestenbrand, Germany, is used.

A second dry-etch step has to be employed for etching into the cladding in the area of the film-waveguide lens. The replacement of the original cylindrical mirror (the curved semiconductor facet) by the combination of the cleaved plane facet with the dry-etched film-waveguide lens is done for technological reasons: for the hypothetic case with a curved (nonoblique) mirror the curvature would have to be so small (the radius of curvature $R$ so large) that the distance along the optical axis between the mirror edges and the center of the mirror would be around $225 \mathrm{~nm}$ only, which is not suitable for standard photolithography.

The desired etch-depth for the waveguide lens has to be hit with an accuracy of better than $25 \mathrm{~nm}$. Otherwise the focal length of the waveguide lens would differ from the resonator length by more than $30 \%$ [28], resulting in nonexact 1D spatial Fourier-transformation and nonoptimal spatial filtering.

In order to have an in situ (real-time) etch-depth control with this demanding accuracy we have successfully employed an adequate measurement techniques, by transferring the concept of reflectance anisotropy spectroscopy (RAS), which is well-known from epitaxy for growth control by now [29-35], to reactive ion etching (RIE) of monocrystalline semiconductor layer sequences [36-39]. We have used argon as the plasma gas and 2 volume- $\%$ of chlorine as the reactive gas. This way the RIE-RAS signals and signal transients have been meaningful and we have achieved an accuracy in in situ etch-depth control of $16 \mathrm{~nm}$. (In cases with low etch rates (below $\approx 75 \mathrm{~nm} / \mathrm{min}$ ) we have even resolved monolayer etch ablation [39].) The details of the RIE-RAS principle and of its experimental results during film-waveguide etching are given elsewhere $[28,38]$. 


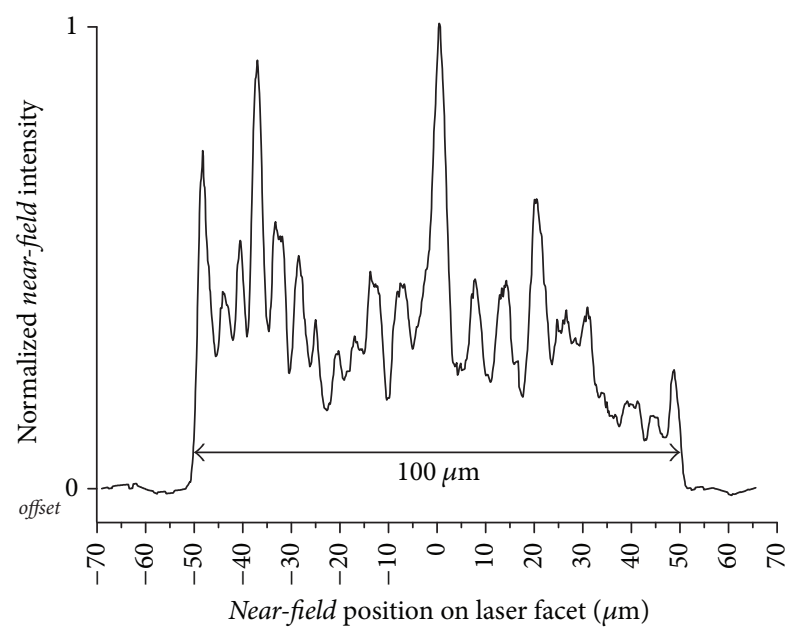

(a)

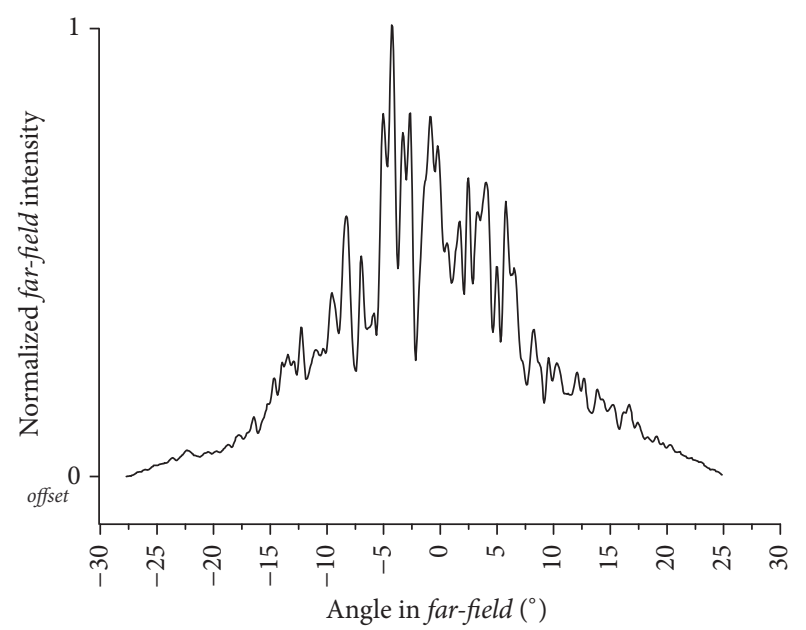

(b)

FIGURE 4: Experimental results, that is, near- (a) and far-field (b) intensity distributions, for a BAL without spatial filter or waveguide lens for com parison. The laser has exactly the same layer sequence as that with the results in Figure 5, since both lasers have been made from the same wafer. The measurements are performed at $91 \mathrm{~K}$ device temperature in continuous wave operation. As expected there is no evidence of fundamental transverse mode selection (TMS\#0).

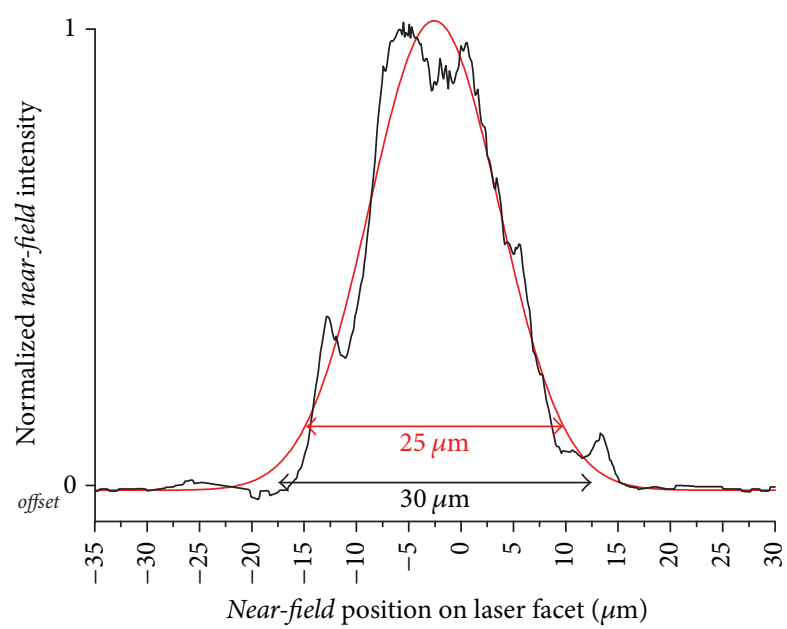

(a)

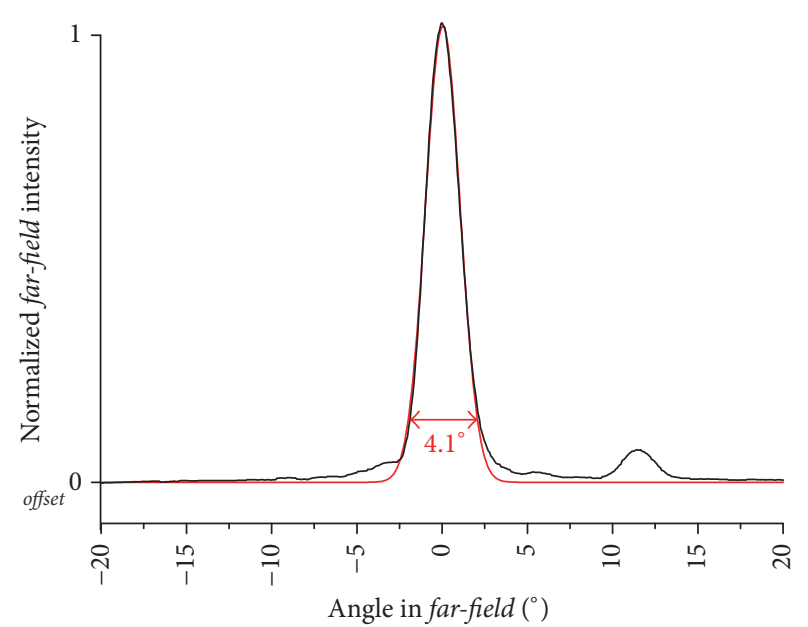

(b)

FIGURE 5: Experimental results for a TMS\#0-BAL with spatial filter to support the fundamental transverse mode (\#0) and with film-waveguide lens. The intensity profiles are taken on the filter side of the resonator. The laser has exactly the same layer sequence as that from Figure 4 , since both lasers have been made from the same wafer. Again (a) gives the near-field, (b) the far-field intensity distribution. The measurements are performed at $91 \mathrm{~K}$ device temperature in continuous wave operation. Obviously the fundamental transverse mode is strongly supported. But filamentation is not suppressed. Gaussian fits are introduced into the diagrams as red lines.

\section{Experimental TMS Results and Discussion}

The devices show clear laser characteristics. The best specimen emits an optical power of about $10 \mathrm{~mW} /$ facet continuous wave at room temperature and at a pump current of $20 \%$ above the laser threshold current $I_{\text {th }}$. This very sample shows a good differential quantum efficiency of $\eta=2.5 \%$.

The TMS measurements were performed at a device temperature of $91 \mathrm{~K}$. To illustrate the TMS results the emission of two BAL devices made from the same wafer (identical layer sequences) will be compared. In Figure 4 the near- and far-field intensity distributions are reproduced for the case neither with a spatial frequency filter nor with a waveguide lens. It is obvious from the results depicted in Figure 4 that there is no transverse mode selection at all, as expected.

On the contrary, in Figure 5 the corresponding near- and far-field intensity distributions are given for a TMS\#0-BAL, that is, a laser with spatial frequency filter for mode \#0 and 
film-waveguide lens. The intensity profiles are taken on the filter side of the resonator. The fundamental transverse mode (\#0 with one intensity maximum) is strongly supported.

But dips and bumps in the envelope of the near-field intensity distribution are discernible as compared to the Gaussian fit (red line). In principle they might either be related to the additional (weak) oscillation of an undesired higher transverse mode (i.e., \#4 with five intensity maxima here) or the occurrence of filamentation [40-45]. But the filter plane also serves as the image plane of the $4 f$ set-up, where, due to the $30 \mu \mathrm{m}$ narrow central segment, intensity maxima of potentially oscillating higher order transverse modes are stripped, inevitably resulting in suppression of these modes. Thus the deviations from the desired smooth envelope are likely to be attributable to filamentation.

The intensity distributions allow for calculation of a $1 \mathrm{D}$ beam quality parameter, that is,

$$
M_{1 \mathrm{D}}^{2}=\frac{\pi w_{0} \theta_{1 \mathrm{D}}}{\lambda_{0}}=1.47
$$

with a full far-field angle of $2 \theta_{1 \mathrm{D}}=4.1^{\circ}$. These (best) results are taken at $10 \%$ above laser threshold, but similar values are achieved for pump currents of up to $20 \%$ above threshold. At pump currents more than $25 \%$ above threshold TMS is not observed.

\section{Conclusions}

A concept for fundamental spatial transverse mode selection (TMS\#0) of edge-emitting broad area (semiconductor diode) lasers (BAL) is presented, which employs a twiceretracted $4 f$ set-up with an actual length of $1 f$ integrated into the laser resonator. One resonator facet incorporates the spatial frequency filter; the other one houses a plane facet in combination with a film-waveguide lens as the Fouriertransform element. Experimental results show good fundamental transverse mode selection for pump currents of up to $20 \%$ above threshold, but filamentation is not suppressed. The best one-dimensional beam quality parameter measured is $M_{1 \mathrm{D}}^{2}=1.47$.

\section{Conflicts of Interest}

The authors declare that they have no conflicts of interest.

\section{Acknowledgments}

This research has been funded by the German Research Foundation (Deutsche Forschungsgemeinschaft, DFG) under Contracts FO 157/44 and FO 157/46. Technological assistance by the Nano Structuring Center (NSC) of the University of Kaiserslautern is gratefully acknowledged.

\section{References}

[1] K. Shigihara, Y. Nagai, S. Kakimoto, and K. Ikeda, "Achieving Broad-Area Laser Diodes with High Output Power and SingleLobed Far-Field Patterns in the Lateral Direction by Loading a Modal Reflector," IEEE Journal of Quantum Electronics, vol. 30, no. 8, pp. 1683-1690, 1994.

[2] J. P. Hohimer, G. R. Hadley, and A. Owyoung, "Mode control in broad-area diode lasers by thermally induced lateral index tailoring," Applied Physics Letters, vol. 52, no. 4, pp. 260-262, 1988.

[3] H. Wenzel, P. Crump, J. Fricke, P. Ressel, and G. Erbert, "Suppression of higher-order lateral modes in broad-area diode lasers by resonant anti-guiding," IEEE Journal of Quantum Electronics, vol. 49, no. 12, pp. 1102-1108, 2013.

[4] J. P. Leidner and J. R. Marciante, "Beam quality improvement in broad-area semiconductor lasers via evanescent spatial filtering," IEEE Journal of Quantum Electronics, vol. 48, no. 10, pp. 1269-1274, 2012.

[5] M. Winterfeldt, P. Crump, S. Knigge, A. Maaßdorf, U. Zeimer, and G. Erbert, "High Beam Quality in Broad Area Lasers via Suppression of Lateral Carrier Accumulation," IEEE Photonics Technology Letters, vol. 27, no. 17, pp. 1809-1812, 2015.

[6] J. Rong, E. Xing, Y. Zhang et al., "Low lateral divergence 2 $\mu \mathrm{m}$ InGaSb/AlGaAsSb broad-area quantum well lasers," Optics Express, vol. 24, no. 7, pp. 7246-7252, 2016.

[7] C. Zink, M. Niebuhr, A. Jechow, A. Heuer, and R. Menzel, "Broad area diode laser with on-chip transverse Bragg grating stabilized in an off-axis external cavity," Optics Express, vol. 22, no. 12, pp. 14108-14113, 2014.

[8] M. Niebuhr, C. Zink, A. Jechow, A. Heuer, L. B. Glebov, and R. Menzel, "Mode stabilization of a laterally structured broad area diode laser using an external volume Bragg grating," Optics Express, vol. 23, no. 9, pp. 12394-12400, 2015.

[9] C. Simmendinger, D. Preier, and O. Hess, "Stabilization of chaotic spatiotemporal filamentation in large broad area lasers by spatially structured optical feedback," Optics Express, vol. 5, no. 3, pp. 48-54, 1999.

[10] N. McCarthy, S. Mailhot, and Y. Champagne, "Single-mode operation of a broad-area semiconductor laser with an anamorphic external cavity: Experimental and numerical results," Applied Optics, vol. 39, no. 7-36, pp. 6806-6813, 2000.

[11] S. Wolff and H. Fouckhardt, "Intracavity stabilization of broad area lasers by structured delayed optical feedback," Optics Express, vol. 7, no. 6, pp. 222-227, 2000.

[12] V. Raab and R. Menzel, "External resonator design for highpower laser diodes that yields $400 \mathrm{~mW}$ of TEM00 power," Optics Expresss, vol. 27, no. 3, pp. 167-169, 2002.

[13] S. K. Mandre, I. Fischer, and W. Elsässer, "Control of the spatiotemporal emission of a broad-area semiconductor laser by spatially filtered feedback," Optics Expresss, vol. 28, no. 13, pp. 1135-1137, 2003.

[14] S. Wolff, A. Rodionov, V. E. Sherstobitov, and H. Fouckhardt, "Fourier-optical transverse mode selection in external-cavity broad-area lasers: experimental and numerical results," IEEE Journal of Quantum Electronics, vol. 39, no. 3, pp. 448-458, 2003.

[15] T. Heil, I. Fischer, and W. Elsaesser, "Coexistence of lowfrequency fluctuations and stable emission on a single high-gain mode in semiconductor lasers with external optical feedback," Physical Review A: Atomic, Molecular and Optical Physics, vol. 58, no. 4, pp. R2672-R2675, 1998.

[16] F. Rogister, P. Mégret, O. Deparis, and M. Blondel, "Coexistence of in-phase and out-of-phase dynamics in a multimode external-cavity laser diode operating in the low-frequency fluctuations regime," Physical Review A: Atomic, Molecular and Optical Physics, vol. 62, article 061803, no. 6, 4 pages, 2000. 
[17] J. M. Buldú, J. García-Ojalvo, and M. C. Torrent, "Delay-induced resonances in an optical system with feedback," Physical Review E: Statistical, Nonlinear, and Soft Matter Physics, vol. 69, Article ID 046207, pp. 046207-046212, 2004.

[18] S. K. Mandre, I. Fischer, and W. Elsäßer, "Spatiotemporal emission dynamics of a broad-area semiconductor laser in an external cavity: Stabilization and feedback-induced instabilities," Optics Communications, vol. 244, no. 1-6, pp. 355-365, 2005.

[19] S. Wolff, C. Doering, A. Rodionov, V. E. Sherstobitov, and H. Fouckhardt, "Self-pulsation in broad area lasers with transverse-mode selective feedback," Optics Communications, vol. 265, no. 2, pp. 642-648, 2006.

[20] K.-H. Hasler, B. Sumpf, P. Adamiec et al., "5-W DBR tapered lasers emitting at $1060 \mathrm{~nm}$ with a narrow spectral linewidth and a nearly diffraction-limited beam quality," IEEE Photonics Technology Letters, vol. 20, no. 19, pp. 1648-1650, 2008.

[21] C. Fiebig, G. Blume, C. Kaspari et al., "12W high-brightness single-frequency DBR tapered diode laser," IEEE Electronics Letters, vol. 44, no. 21, pp. 1253-1255, 2008.

[22] M. Spreemann, M. Lichtner, M. Radziunas, U. Bandelow, and H. Wenzel, "Measurement and simulation of distributed-feedback tapered master-oscillator power amplifiers," IEEE Journal of Quantum Electronics, vol. 45, no. 6, pp. 609-616, 2009.

[23] I. Sergachev, R. Maulini, A. Bismuto, S. Blaser, T. Gresch, and A. Muller, "Gain-guided broad area quantum cascade lasers emitting $23.5 \mathrm{~W}$ peak power at room temperature," Optics Express, vol. 24, no. 17, pp. 19063-19071, 2016.

[24] D. Hoffmann, K. Huthmacher, C. Döring, and H. Fouckhardt, "Broad area lasers with monolithically integrated transverse mode selector," Applied Physics Letters, vol. 96, no. 18, Article ID 181104, 2010.

[25] D. Hoffmann, K. Huthmacher, C. Doering, and H. Fouckhardt, "Broad area lasers with folded-resonator geometry for integrated transverse mode selection," in Proceedings of the Novel In-Plane Semiconductor Lasers X, USA, January 2011.

[26] L. A. Coldren and S. W. Corzine, Diode Lasers and Photonic Integrated Circuits, John Wiley \& Sons, Inc., Hoboken, NJ, USA, 2012.

[27] T. H. Loeber, D. Hoffmann, and H. Fouckhardt, "Dense lying self-organized gaassb quantum dots on GaAs for efficient lasers," Beilstein Journal of Nanotechnology, vol. 2, no. 1, pp. 333338, 2011.

[28] A.-K. Kleinschmidt, Resonatorinterne Transversalmodenselektion bei Antimonid-Breitstreifenlasern [Ph.D. thesis], Physics Department, Technical University Kaiserslautern, Germany, 2017.

[29] D. E. Aspnes, J. P. Harbison, A. A. Studna, and L. T. Florez, "Optical reflectance and electron diffraction studies of molecular-beam-epitaxy growth transients on GaAs(001)," Physical Review Letters, vol. 59, no. 15, pp. 1687-1690, 1987.

[30] D. E. Aspnes, J. P. Harbison, A. A. Studna, and L. T. Florez, "Application of reflectance difference spectroscopy to molecular-beam epitaxy growth of GaAs and AlAs," Journal of Vacuum Science \& Technology A, vol. 6, no. 3, pp. 1327-1332, 1988.

[31] J. P. Harbison, D. E. Aspnes, A. A. Studna, and L. T. Florez, "Optical reflectance measurements of transients during molecular-beam epitaxial growth on (001) GaAs," Journal of Vacuum Science \& Technology B: Microelectronics and Nanometer Structures, vol. 6, no. 2, pp. 740-742, 1988.

[32] Z. Sobiesierski, D. I. Westwood, and C. C. Matthai, "Aspects of reflectance anisotropy spectroscopy from semiconductor surfaces," Journal of Physics: Condensed Matter, vol. 10, no. 1, pp. $1-43,1998$.

[33] J.-T. Zettler, K. Haberland, M. Zorn et al., "Real-time monitoring of MOVPE device growth by reflectance anisotropy spectroscopy and related optical techniques," Journal of Crystal Growth, vol. 195, no. 1-4, pp. 151-162, 1998.

[34] K. Haberland, O. Hunderi, M. Pristovsek, J.-T. Zettler, and W. Richter, "Ellipsometric and reflectance-anisotropy measurements on rotating samples," Thin Solid Films, vol. 313-314, pp. 620-624, 1998.

[35] A. I. Shkrebtii, N. Esser, W. Richter et al., "Reflectance anisotropy of GaAs(100): Theory and experiment," Physical Review Letters, vol. 81, no. 3, pp. 721-724, 1998.

[36] L. Barzen, J. Richter, H. Fouckhardt, M. Wahl, and M. Kopnarski, "Monitoring of (reactive) ion etching (RIE) with reflectance anisotropy spectroscopy (RAS) equipment," Applied Surface Science, vol. 328, pp. 120-124, 2015.

[37] L. Barzen, A.-K. Kleinschmidt, J. Strassner et al., "Influence of plasma composition on reflectance anisotropy spectra for in situ III-V semiconductor dry-etch monitoring," Applied Surface Science, vol. 357, pp. 530-538, 2015.

[38] A. Kleinschmidt, L. Barzen, J. Strassner et al., "Precise in situ etch depth control of multilayered III-V semiconductor samples with reflectance anisotropy spectroscopy (RAS) equipment," Beilstein Journal of Nanotechnology, vol. 7, pp. 1783-1793, 2016.

[39] C. Doering, A.-K. Kleinschmidt, L. Barzen, J. Strassner, and H. Fouckhardt, "Atomic layer sensitive in-situ plasma etch depth control with reflectance anisotropy spectroscopy (RAS)," in Proceedings SPIE 10329 Optical Measurement Systems for Industrial Inspection X, Munich, Germany, 2017.

[40] A. H. Paxton and G. C. Dente, "Filament formation in semiconductor laser gain regions," Journal of Applied Physics, vol. 70, no. 6, pp. 2921-2925, 1991.

[41] J. R. Marciante and G. P. Agrawal, "Spatio-temporal characteristics of filamentation in broad-area semiconductor lasers," IEEE Journal of Quantum Electronics, vol. 33, no. 7, pp. 1174-1179, 1997.

[42] J. R. Marciante and G. P. Agrawal, "Spatio-temporal characteristics of filamentation in broad-area semiconductor lasers: Experimental results," IEEE Photonics Technology Letters, vol. 10, no. 1, pp. 54-56, 1998.

[43] J. R. Marciante and G. P. Agrawal, "Nonlinear mechanisms of filamentation in broad-area semiconductor lasers," IEEE Journal of Quantum Electronics, vol. 32, no. 4, pp. 590-596, 1996.

[44] O. Hess, S. W. Koch, and J. V. Moloney, "Filamentation and Beam Propagation in Broad-Area Semiconductor Lasers," IEEE Journal of Quantum Electronics, vol. 31, no. 1, pp. 35-43, 1995.

[45] M. Winterfeldt, P. Crump, S. Knigge, A. Maaßdorf, G. Erbert, and G. Tränkle, "The Influence of differential modal gain on the filamentary behavior of broad area diode lasers," in Proceedings of the IEEE Photonics Conference, IPC 2015, pp. 567-568, USA, August 2015. 


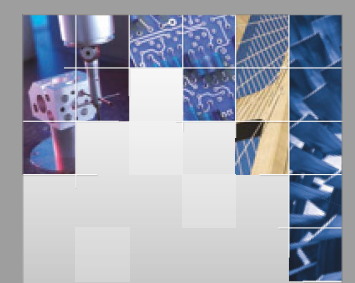

\section{Enfincering}
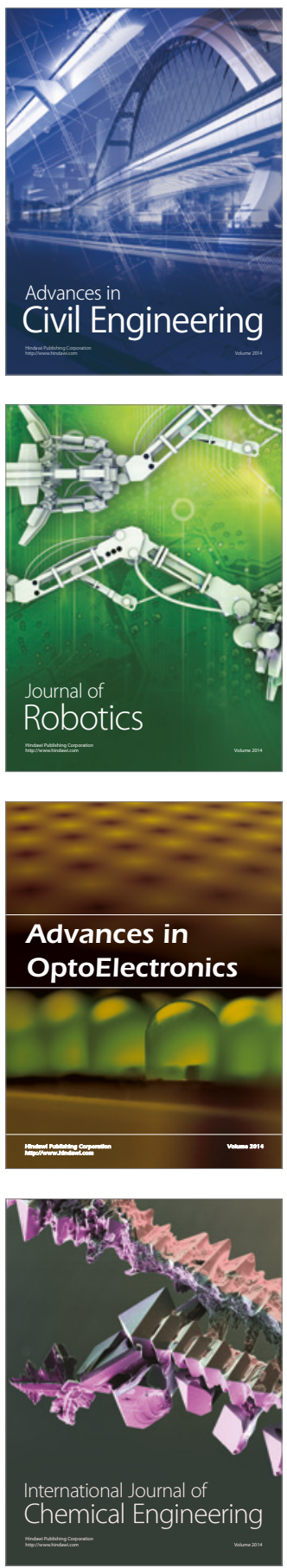

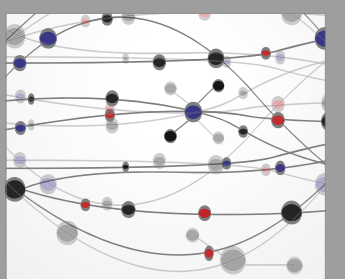

The Scientific World Journal

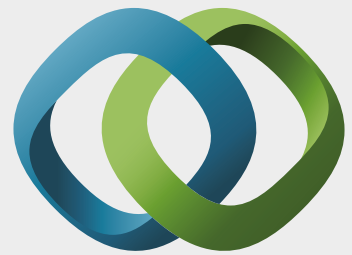

\section{Hindawi}

Submit your manuscripts at

https://www.hindawi.com
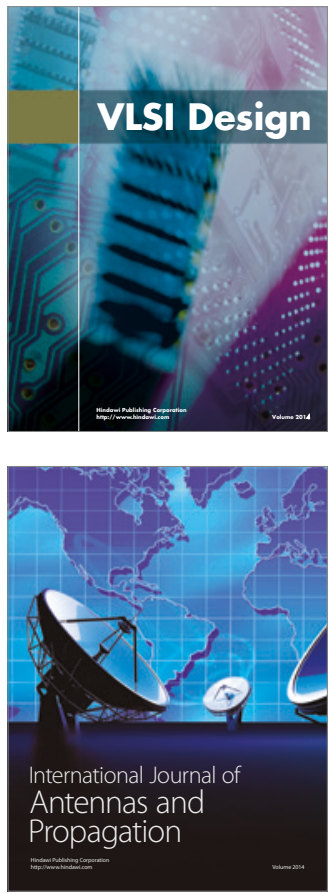

\section{Rotating}

Machinery
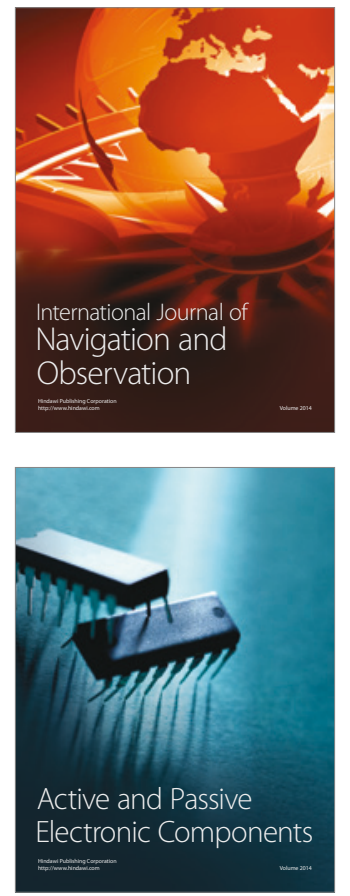
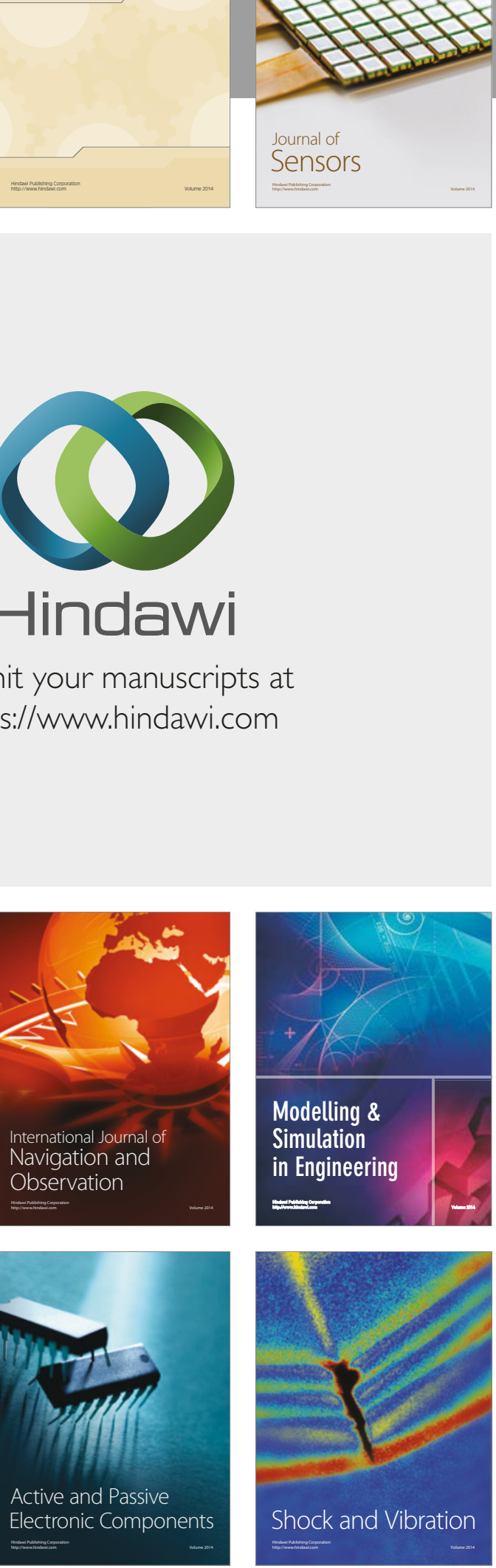
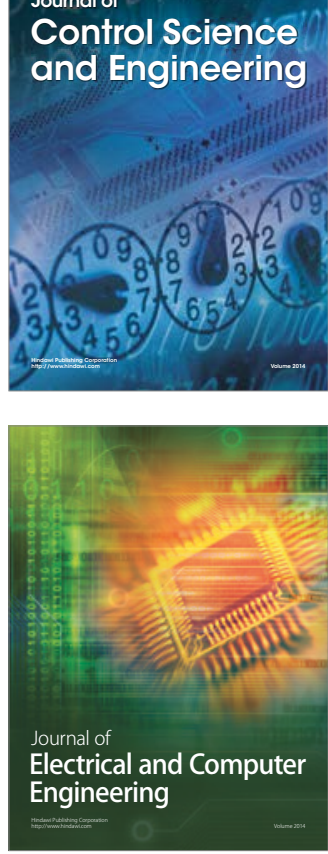

Distributed

Journal of

Control Science

and Engineering
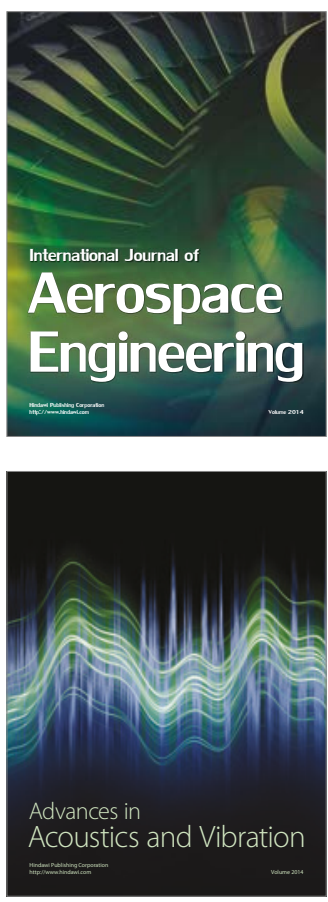

Sensor Networks 